\section{METABOLISM}

\section{REG $\gamma$ ulating lipid metabolism}

A new study uncovers a link between the proteasome and autophagy in lipid metabolism and identifies the ubiquitin-independent REG $\gamma$ proteasome system as a key metabolic regulator (Cell Metab. 18, 380-391).

Shuxian Dong et al. showed that knocking out the REG $\gamma$ proteasome in mice led to autophagy and could protect mice from highfat diet (HFD)-induced lipid steatosis in the liver. Delving into the underlying mechanism for the effects of REG $\gamma$, the authors found that in normal conditions it binds sirtuin 1 (SirT1), a protein deacetylase that has been described to alter lifespan and cellular metabolism. REG $\gamma$ binding of SirT1 promotes ubiquitinindependent degradation of SirT1. This displaces the autophagy-related Atg proteins from SirT1 and increases their acetylation, thus inhibiting autophagy. In conditions of energy deprivation, REG $\gamma$ dissociates from SirT1, thus allowing SIRT1 to interact with its Atg targets and upregulate autophagy.

The researchers then showed that treating HFD-fed REG $\gamma$-knockout mice with an autophagy inhibitor increased lipid droplet accumulation in the liver to similar levels to as in HFD-fed wild-type mice, suggesting that autophagy is required for the protection against hepatic steatosis elicited by knocking out REG $\gamma$.

This identification of $R E G \gamma$ as a regulator of autophagy and lipid metabolism could lead to approaches to target REG $\gamma$ for therapeutic benefit in a diverse range of metabolic disorders. $-M S$

\section{DISEASES OF THE NERVOUS SYSTEM}

\section{The complexity of compensation}

Tuberous sclerosis complex (TSC) is a neurodevelopmental disorder characterized by autism and epilepsy and is caused by mutations in one of the two TSC genes (TSC1 and TSC2). Now, Wyatt B. Potter et al. report that aberrant signaling through the glutamate receptor mGluR5 is responsible for the dysfunction in synaptic plasticity that causes the neurological manifestations of the disease (PLOS Biol. 11, e1001627).

Changes in synaptic strength occur during learning and development. But when this synaptic plasticity does not occur normally, it can result in epilepsy or in behavioral inflexibility because of the inability to unlearn a behavior.

\title{
BONE METASTASES
}

\section{Bad to the bone}

A new report delineates how the primary tumor stroma may preselect breast cancer cells that have specific metastatic tropism for bone (Cell 154, 1060-1073).

Breast cancers are prone to metastasizing to bone, among other organs. Now, Xiang Zhang et al. show that interactions between cancer cells and the primary tumor stroma may enrich for tumor cells better fitted to survive and colonize specific tissues such as bone.

Previous research had shown that activation of SRC signaling in primary breast tumors, independent of their subtype, correlates with bone metastasis. In this report, the authors focused on a subset of triple-negative breast tumors, which show metastatic tropism for bone and in which SRC activation is not linked to a driver oncogenic event.

The authors studied how cells that have activated SRC signaling are enriched in some primary tumors prior to metastatic spread, and they found that signals from the breast stroma can exert a positive selection for this trait. Cancer-associated fibroblasts (CAFs) were enriched in the environment of bone-tropic triple-negative breast tumors, where they secreted the factors $\mathrm{C}-\mathrm{X}-\mathrm{C}$ motif chemokine 12 and insulin-like growth factor 1 . SRC activation enhances the effects of these cytokines on downstream signaling pathways that promote survival and growth of tumor cells. Interestingly, the researchers found that these cytokines are also enriched in the bone marrow stroma of triple-negative tumors. The cytokine-secreting CAFs in the primary tumor stroma can confer a growth advantage to cells with activated SRC signaling from the tumor milieu that primes them for survival and growth in the secondary bone environment.

The findings add a layer of complexity to the exquisite regulation of metastatic organotropism and shed light on how it can be selected for before spread occurs, further paving the way for potential therapeutic interventions early in metastasis. - VA

Synaptic plasticity has previously been reported to be reduced in juvenile Tsc2deficient mice. Potter et al. found that it is then restored in adult Tsc2-deficient mice, but that this is as a result of aberrant compensatory upregulation of the expression and activity of mGluR5. This enhanced mGluR5 activity had a role in the epileptic and autistic phenotypes in Tsc2-deficient mice: an mGluR5 antagonist could reduce epileptic activity in the brains of the mice and could prevent their autistic-like perseverative behaviors. These findings suggest that inhibiting mGluR5 could be a potential therapeutic approach to treat TSC in humans. - EC

\section{INFLAMMATORY DISEASES}

\section{Blocking lymphocyte access}

Two clinical trials show that vedolizumab, an antibody against integrin $\alpha_{4} \beta_{7}$ that selectively blocks lymphocyte trafficking into the gut, provides therapeutic benefit to people with ulcerative colitis or Crohn's disease (N. Eng/. J. Med. 369, 699-710 and 711-721).

The first study, authored by Brian Feagan et al., tested vedolizumab in nearly 900 patients with ulcerative colitis. The second one, by William Sandborn et al., involved over 1,100 patients with Crohn's disease. Both placebo-controlled trials were designed to test whether the antibody had any effect in inducing an initial response and as maintenance therapy against the two diseases.

In the ulcerative colitis trial, $47 \%$ of vedolizumab-treated patients showed a clinical response at week 6 of the study, compared to a $26 \%$ response rate in people who received placebo. Among patients who carried on receiving the antibody during the maintenance phase of the trial, $45 \%$ were in remission at week 52 , compared to $16 \%$ of the patients who switched to placebo. In the Crohn's trial, vedolizumab failed to induce a response in the induction 